\title{
Towards Making the Most of BERT in Neural Machine Translation
}

\author{
Jiacheng Yang, ${ }^{1}$ Mingxuan Wang, ${ }^{2}$ Hao Zhou, ${ }^{2}$ Chengqi Zhao, ${ }^{2}$ Weinan Zhang, ${ }^{1}$ Yong Yu, ${ }^{1}$ Lei Li ${ }^{2}$ \\ ${ }^{1}$ Shanghai Jiao Tong University \\ ${ }^{2}$ ByteDance AI Lab, Beijing, China \\ \{PrayStarJirachi, wnzhang, yyu\}@sjtu.edu.cn, \\ \{wangmingxuan.89, zhouhao.nlp, zhaochengqi.d, lileilab\}@ bytedance.com
}

\begin{abstract}
GPT-2 and BERT demonstrate the effectiveness of using pretrained language models (LMs) on various natural language processing tasks. However, LM fine-tuning often suffers from catastrophic forgetting when applied to resource-rich tasks. In this work, we introduce a concerted training framework (CTNMT) that is the key to integrate the pre-trained LMs to neural machine translation (NMT). Our proposed CTNMT consists of three techniques: $a$ ) asymptotic distillation to ensure that the NMT model can retain the previous pre-trained knowledge; $b$ ) a dynamic switching gate to avoid catastrophic forgetting of pre-trained knowledge; and $c$ ) a strategy to adjust the learning paces according to a scheduled policy. Our experiments in machine translation show CTNMT gains of up to 3 BLEU score on the WMT14 English-German language pair which even surpasses the previous state-of-the-art pretraining aided NMT by 1.4 BLEU score. While for the large WMT14 English-French task with 40 millions of sentencepairs, our base model still significantly improves upon the state-of-the-art Transformer big model by more than 1 BLEU score.
\end{abstract}

\section{Introduction}

Pre-trained text representations like ELMo (Peters et al. 2018), GPT-2 (Radford et al. 2019; 2018) and BERT (Devlin et al. 2018) have shown their superiors, which significantly boost the performances of various natural language processing tasks, including classification, POS tagging, and question answering. Empirically, on most downstream NLP tasks, fine-tuning BERT parameters in training achieves better results compared to using fixed BERT as features.

However, introducing BERT to neural machine translation (NMT) is non-trivial, directly using BERT in NMT does not always yield promising results, especially for the resource-rich setup. As in many other NLP tasks, we could use BERT as the initialization of NMT encoder, or even directly replace the word embedding layer of the encoderdecoder framework with the BERT embeddings. This does work in some resource-poor NMT scenarios but hardly gives inspiring results in high resource NMT benchmarks such as WMT14 English-French, which always have a large size of parallel data for training. Furthermore, Edunov, Baevski,

Copyright (c) 2020, Association for the Advancement of Artificial Intelligence (www.aaai.org). All rights reserved. and Auli (2019) observe that using pre-trained model in such a way leads to remarkable improvements without finetuning, but give few gains in the setting of fine-tuning in resource-poor scenario. While the gain diminishes when more labeled data become available. This is not in line with our expectation.

We argue that current approaches do not make the most use of BERT in NMT. Ideally, fine-tuning BERT in NMT should lead to adequate gain as in other NLP tasks. However, compared to other tasks working well with direct BERT finetuning, NMT has two distinct characteristics, the availability of large training data (10 million or larger) and the high capacity of baseline NMT models (i.e. Transformer). These two characteristics require a huge number of updating steps during training in order to fit the high-capacity model well on massive data ${ }^{1}$. Updating too much leads to the catastrophic forgetting problem (Goodfellow et al. 2013), namely too much updating in training make the BERT forget its universal knowledge from pre-training. The assumption lies well with previous observations that fixed BERT improves NMT a bit and fine-tuning BERT even offers no gains.

In this paper, we propose the concerted training approach (CTNMT) to make the most use of BERT in NMT. Specifically, we introduce three techniques to integrate the power of pre-trained BERT and vanilla NMT, namely asymptotic distillation, dynamic switch for knowledge fusion, and ratescheduled updating. First, an asymptotic distillation (AD) technique is introduced to keep remind the NMT model of BERT knowledge. The pre-trained BERT serves as a teacher network while the encoder of the NMT model serves as a student. The objective is to mimic the original teacher network by minimizing the loss (typically L 2 or cross-entropy loss) between the student and the teacher in an asymptotic way. The asymptotic distillation does not introduce additional parameters therefore it can be trained efficiently. Secondly, a dynamic switching gate (DS) is introduced to combine the encoded embedding from BERT and the encoder of NMT. Based on the source input sentence, it provides an adaptive way to fuse the power of BERT and NMT's encoder-decoder network. The intuition is that for some

\footnotetext{
${ }^{1}$ For example, for the EN-DE translation task, it always takes 100 thousands of training steps, while a typical POS tagging model needs several hundreds of steps.
} 
source sentences BERT might produce a better encoded information than NMT's encoder while it is opposite for other sentences. Thirdly, we develop a scheduling policy to adjust the learning rate during the training. Without such a technique, traditionally BERT and NMT are updated uniformly. However, a separate and different updating pace for BERT LM is beneficial for the final combined model. Our proposed rate-scheduled learning effectively controls the separate paces of updating BERT and NMT networks according to a policy. With all these techniques combined, CTNMT empirically works effectively in machine translation tasks.

While both simple and accurate, Our experiments in English-German, English-French, and English-Chinese show gains of up to 2.9, 1.3 and 1.6 BLEU score respectively. The results even surpass the previous state-of-the-art pre-training aided NMT by +1.4 BLEU score on the WMT English-German benchmark dataset. It is also worth mentioning that the improvements not comes from the additional parameters, CTNMT significantly bypass a strong MultiCol deep NMT model which is pretrained with the bilingual corpus by 1.4 and 0.6 BLEU respectively(Chen et al. 2018).

The main contributions of our work can be summarized as:

- We are the first to investigate the catastrophic forgetting problem on the NMT context when incorporating large language models;

- We propose CTNMT to alleviate the problem. CTNMT can also be applied to other NLP tasks;

- We make the best practice to utilize the pre-trained model. Our experiments on the large scale benchmark datasets show significant improvement over the state-of-the-art Transformer-big model.

\section{The Proposed CTnMT}

As can be seen in Figure 1, we will describe CTNMT to modify sequence to sequence learning to effectively utilize the pre-trained LMs.

\section{Background}

Sequence modeling in machine translation has been largely focused on supervised learning which generates a target sentence word by word from left to right, denoted by $p_{\theta}(Y \mid X)$, where $X=\left\{x_{1}, \cdots, x_{m}\right\}$ and $Y=\left\{y_{1}, \cdots, y_{n}\right\}$ represent the source and target sentences as sequences of words respectively. $\theta$ is the set of parameters which is usually trained to minimize the negative log-likelihood:

$$
\mathcal{L}_{\mathrm{nmt}}=-\sum_{i=1}^{n} \log p_{\theta}\left(y_{i} \mid y_{<i}, X\right) .
$$

where $m$ and $n$ is the length of the source and the target sequence respectively.

Specifically, the encoder is composed of $L$ layers. The first layer is the word embedding layer and each encoder layer is calculated as:

$$
h_{e}^{l}=\operatorname{Encode}\left(h_{e}^{l-1}\right)
$$

Encoder $(\cdot)$ is the layer function which can be implemented as RNN, CNN, or self-attention network. In this work, we evaluate CTNMT on the standard Transformer model, while it is generally applicable to other types of NMT architectures.

The decoder is composed of $L$ layers as well:

$$
h_{d}^{l}=\operatorname{Decoder}\left(h_{e}^{L}, h_{d}^{l-1}\right)
$$

which is calculated based on both the lower decoder layer $h_{d}^{l-1}$ and the top-most encoder layer $h_{e}^{L}$. The last layer of the decoder $h_{d}^{L}$ is used to generate the final output sequence. Without the encoder, the decoder essentially acts as a language model on $y$ 's. Similarly, the encoder with an additional output layer also serves as a language model. Thus it is natural to transfer the knowledge from the pre-trained languages models to the encoder and decoder of NMT.

Without adjusting the actual language model parameters, BERT and GPT-2 form the contextualized word embedding based on language model representations. GPT-2 can be viewed as a causal language modeling (CLM) task consisting of a Transformer LM trained to fit the probability of a word given previous words in a sentence, while BERT is designed to pre-train deep bidirectional representations by jointly conditioning on both left and right context in all layers. Specifically, from an input sentence $X=\left\{x_{1} \cdots, x_{m}\right\}$, BERT or GPT-2 computes a set of feature vectors $H^{l m}=$ $\left\{h_{1}^{l m} \ldots, h_{m}^{l m}\right\}$ upon which we build our NMT model. In general, there are two ways of using BERT features, namely fine-tuning approach, and feature approach. For fine-tuning approach, a simple classification layer is added to the pretrained model and all parameters are jointly fine-tuned on a downstream task, while the feature approach keeps the pretrained parameters unchanged. For most cases, the performance of the fine-tuning approach is better than that of the feature approach.

In NMT scenario, the basic procedure is to pre-train both the NMT encoder and decoder networks with language models, which can be trained on large amounts of unlabeled text data. Then following a straightforward way to initialize the NMT encoder with the pre-trained LM and fine-tune with a labeled dataset. However, this procedure may lead to catastrophic forgetting, where the model performance on the language modeling tasks falls dramatically after finetuning (Goodfellow et al. 2013). With the increasing training corpus, the benefits of the pre-training will be gradually diminished after several iterations of the fine-tuning procedure. This may hamper the model's ability to utilize the pretrained knowledge. To tackle this issue, we propose three complementary strategies for fine-tuning the model.

\section{Asymptotic Distillation}

Addressing the catastrophic forgetting problem, we propose asymptotic distillation as the minic regularization to retain the pre-trained information. Additionally, due to the large number of parameters, BERT and GPT-2, for example, cannot be deployed in resource-restricted systems such as mobile devices. Fine-tuning with the large pre-trained model slows NMT throughput during training by about $9.2 \mathrm{x}$, as 


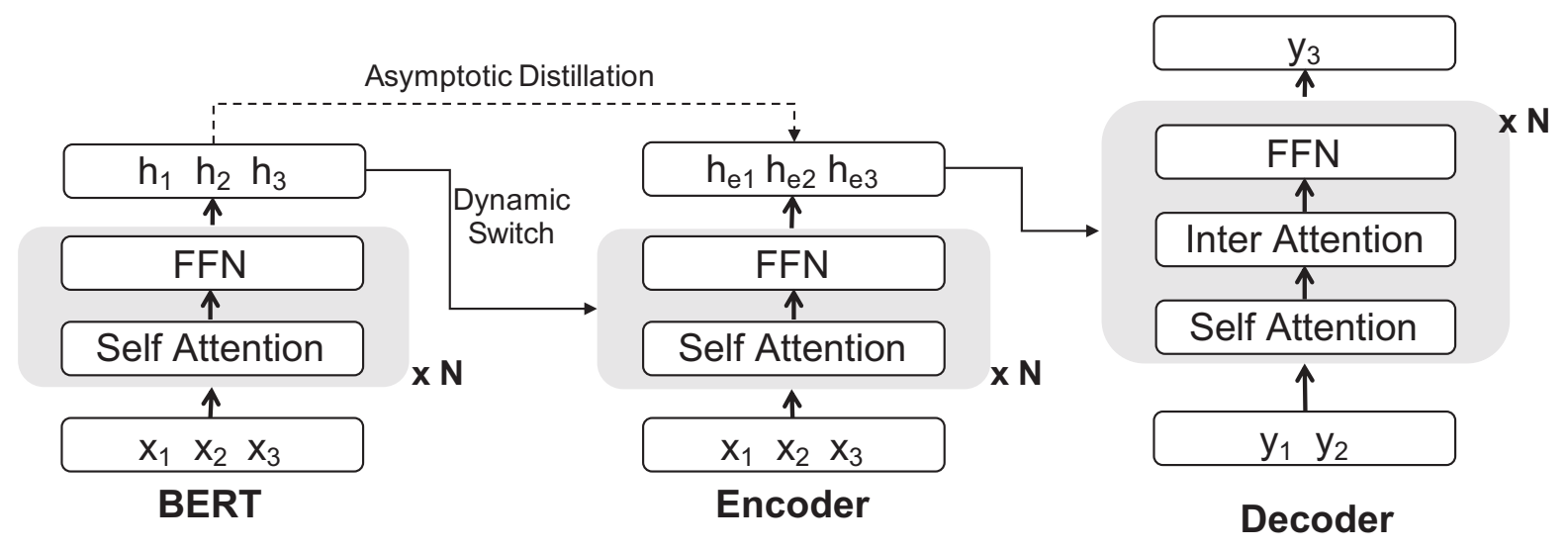

Figure 1: The overall CTNMT with asymptotic distillation and dynamic switch.

showed by (Edunov, Baevski, and Auli 2019). With asymptotic distillation, we can train the NMT model without additional parameters.

Specifically, the distillation objective is to penalize the mean-squared-error (MSE) loss between the hidden states of the NMT model and the pre-trained LM:

$$
\mathcal{L}_{\mathrm{kd}}=-\left\|\hat{h}^{l m}-h_{l}\right\|_{2}^{2}
$$

where the hidden state of the pre-trained language model $\hat{h}^{l m}$ is fixed and treated as the teacher; $h_{l}$ is the $l^{\text {th }}$ layer of the hidden states of the NMT model. For the encoder part, we use the last layer and find it is better to add the supervision signal to the top encoder layers.

At training time for NMT, the distilling objective can be used in conjunction with a traditional cross-entropy loss:

$$
\mathcal{L}=\alpha \cdot \mathcal{L}_{\mathrm{nmt}}+(1-\alpha) \cdot \mathcal{L}_{\mathrm{kd}}
$$

where $\alpha$ is a hyper-parameter that balances the preference between pre-training distillation and NMT objective.

\section{Dynamic Switch}

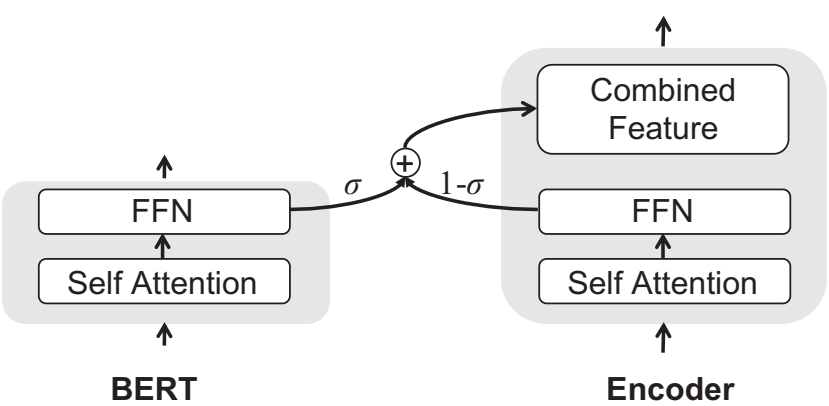

Figure 2: The dynamic switch fashion

Asymptotic distillation provides an effective way to integrate the pre-trained information to NMT tasks. Features extracted from a extremely large pre-trained LM such as
BERT, however, are not easy for the student Transformer network to fit since these features can be high-ordered. Meanwhile, directly feeding the features to the NMT model ignores the information from the original text, which harms the performance. We thus introduce a dynamic switch strategy to incorporate the pre-trained model to the original Transformer NMT model as showed in 2 .

Inspired by the success of gated recurrent units in RNN(Chung et al. 2014), we propose to use the similar idea of gates to dynamically control the amount of information flowing from the pre-trained model as well as the NMT model and thus balance the knowledge transfer for our NMT model.

Intuitively, the context gate looks at the input signals from both the pre-trained model and the NMT model and outputs a number between 0 and 1 for each element in the input vectors, where 1 denotes "completely transferring this" while 0 denotes "completely ignoring this". The corresponding input signals are then processed with an element-wise multiplication before being fed to the next layer. Formally, a context gate consists of a sigmoid neural network layer and an element-wise multiplication operation which is computed as:

$$
g=\sigma\left(W h^{l m}+U h^{n m t}+b\right)
$$

where $\sigma(\cdot)$ is the logistic sigmoid function, $h^{l m}$ is the hidden state of the pre-trained language model, and $h^{n m t}$ is the hidden state of the original NMT. Then, we consider integrating the NMT model and pre-trained language model as:

$$
h=g \odot h^{l m}+(1-g) \odot h^{n m t}
$$

where $\odot$ is an element-wise multiplication. If $g$ is set to 0 , the network will degrade to the traditional NMT model; if $g$ is set to 1 , the network will simply act as the fine-tuning approach.

\section{Rate-scheduled learning}

We also propose a rate-scheduled learning strategy, as an important complement, to alleviate the catastrophic forgetting problem. Instead of using the same learning rate for 


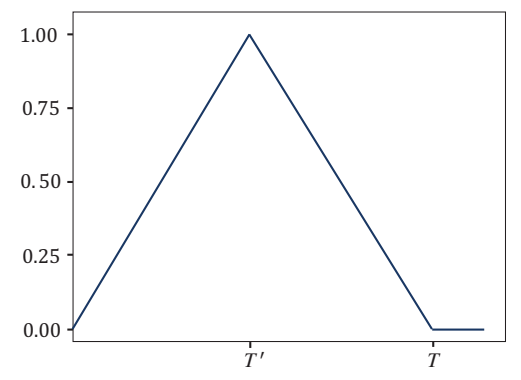

Figure 3: The slanted triangular learning rate schedule used for $\eta^{l m}$.

all components of the model, rate-scheduled learning strategy allows us to tune each component with different learning rates. Formally, the regular stochastic gradient descent (SGD) update of a model's parameters $\theta$ at time step $t$ can be summarized as the following formula:

$$
\theta_{t}=\theta_{t-1}-\eta \nabla_{\theta} \mathcal{L}(\theta)
$$

where $\eta$ is the learning rate. For discriminative fine-tuning, we group the parameters into $\left\{\theta^{l m}, \theta^{n m t}\right\}$, where $\theta^{l m}$ and $\theta^{n m t}$ contain the parameters of the pre-trained language model and the NMT model respectively. Similarly, we obtain the corresponding learning rate $\left\{\eta^{l m}, \eta^{n m t}\right\}$.

The SGD update with drate-scheduled learning strategy is then the following:

$$
\begin{aligned}
\theta_{t}^{l m} & =\theta_{t-1}^{l m}-\eta^{l m} \nabla_{\theta^{l m}} \mathcal{L}\left(\theta^{l m}\right) \\
\theta_{t}^{n m t} & =\theta_{t-1}^{n m t}-\eta^{n m t} \nabla_{\theta^{n m t}} \mathcal{L}\left(\theta^{n m t}\right)
\end{aligned}
$$

We would like the model first to quickly converge the NMT parameters. Then we jointly train both the NMT and LM parameters with modest steps. Finally, we only refine the NMT parameters to avoid forgetting the pre-trained knowledge. Using the same learning rate or an annealed learning rate throughout training is not the best way to achieve this behavior. Inspired by (Howard and Ruder 2018; Smith 2017), we employ slanted triangular learning rates policy which first increases linearly and then decreases gradually after a specified epoch, i.e., there is a "short increase" and a "long decay". More specifically, the learning rate of pre-trained parameters $\eta^{l m}$ is then defined as $\eta^{l m}=\rho \cdot \eta^{n m t}$ where,

$$
\rho= \begin{cases}t / T^{\prime} & t \leq T^{\prime} \\ 1-\frac{t-T^{\prime}}{T-T^{\prime}} & T^{\prime} \leq t<T \\ 0 & t>T .\end{cases}
$$

$T^{\prime}$ is the step after which we switch from increasing to decreasing the learning rate. $T$ is the maximum fine-tuning steps of $\theta^{l m}$ and $t$ is the current training step. We set $T^{\prime}=$ 10000 and $T=20000$ in our experiments. For NMT parameters $\theta^{n m t}$, we generally follow the learning rate strategy described in (Vaswani et al. 2017a).

\section{Experiments Settings}

\section{Datasets}

We mainly evaluate CTNMT on the widely used WMT English-German translation task. In order to show the usefulness of CTNMT, we also provide results on other large-scale translation tasks: English-French, EnglishChinese. The evaluation metric is cased BLEU. We tokenized the reference and evaluated the performance with multi-bleu.pl ${ }^{2}$. The metrics are exactly the same as the previous work (Papineni et al. 2002). All the training and testing datasets are public ${ }^{3}$.

For English-German, to compare with the results reported by previous work, we used the same subset of the WMT 2014 training corpus that contains $4.5 \mathrm{M}$ sentence pairs with 91M English words and 87M German words. The concatenation of news-test 2012 and news-test 2013 is used as the validation set and news-test 2014 as the test set.

We also report the results of English-French. To compare with the results reported by previous work on end-to-end NMT, we used the same subset of the WMT 2014 training corpus that contains $36 \mathrm{M}$ sentence pairs. The concatenation of news-test 2012 and news-test 2013 serves as the validation set and news-test 2014 as the test set.

For English-Chinese, our training data consists of $2.2 \mathrm{M}$ sentence pairs extracted from WMT 2018. We choose WMT 2017 dataset as our development set and WMT 2018 as our test sets.

\section{Training details}

NMT The hyper-parameters setting resembles (Vaswani et al. 2017a). Specifically, we reduce the vocabulary size of both the source language and the target language to $50 \mathrm{~K}$ symbols using the sub-word technique (Bojanowski et al. 2017). During training, we employ label smoothing of value $\epsilon=0.1$ (Pereyra et al. 2017). For strategies using BERT features, we apply the same pre-processing tool as BERT or GPT-2 does to the source language corpus. We batch sentence pairs by approximating length and limited input and output tokens per batch to 8192 per GPU. We train our NMT model with the sentences of length up to 150 words in the training data. We train for 100, 000 steps on 8 V100 GPUs, each of which results in training batch contained approximately $8192 \times 16$ source and target tokens respectively. We use a beam width of 8 and length penalty to 0.6 in all the experiments. For our small model, the dimensions of all the hidden states were set to 768 and for the big model, the dimensions were set to 1024 .

Pre-trained LM For the pre-trained LMs, we apply the public BERT and GPT-2 model to make the experiments reproducible. BERT is a multi-layer, bidirectional transformer encoder that comes in two variants: BERT $_{\mathrm{BASE}}$ and the larger

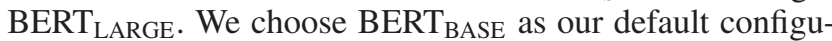
ration which comprises 12 layers, 768 hidden units, 12 selfattention heads, and 110M parameters.

Similarly, OpenAI GPT-2(Radford et al. 2019; 2018) is a generative pre-trained transformer (GPT) encoder fine-tuned on downstream tasks. Unlike BERT, however, GPT-2 is unidirectional and only makes use of the previous context at each time step.

\footnotetext{
${ }^{2}$ https://github.com/moses-smt

${ }^{3}$ http://www.statmt.org/wmt14/translation-task.html
} 


\begin{tabular}{l|l|c|c|c} 
System & Architecture & En-De & En-Fr & En-Zh \\
\hline \hline \multicolumn{3}{|c}{ Existing systems } \\
\hline Vaswani et al. (2017b) & Transformer base & 27.3 & 38.1 & - \\
Vaswani et al. (2017b) & Transformer big & 28.4 & 41.0 & - \\
Lample and Conneau (2019) & Transformer big + Fine-tuning & 27.7 & - & - \\
Lample and Conneau (2019) & Transformer big + Frozen Feature & 28.7 & - & - \\
Chen et al. (2018) & RNMT+ + MultiCol \\
\hline \multicolumn{4}{|c}{ Our NMT systems } \\
\hline CTNMT & Transformer (base) & 28.7 & $41.7-$ & \\
CTNMT & Rate-scheduling & 27.2 & 41.0 & 37.3 \\
CTNMT & Dynamic Switch & 29.7 & 41.6 & 38.4 \\
CTNMT & Asymptotic Distillation & 29.4 & 41.4 & 38.6 \\
CTNMT & + ALL & 29.2 & 41.6 & 38.3 \\
&
\end{tabular}

Table 1: Case-sensitive BLEU scores on English-German, English-French and English-Chinese translation. The best performance comes from the fusion of rate-scheduling, dynamic switch and asymptotic distillation.

Pre-trained LM for NMT Our experiments mainly conducted on the encoder part of BERT. If not specified, we choose the second-to-last hidden states of BERT to help the training of NMT. For rate-scheduled learning in Eq. (10), $T^{\prime}$ is set to 10,000 and $T$ is set to 20,000 and we found the performance is rather robust to the hyper-parameters in our preliminary experiments. For dynamic switch, we make a gate combination of the second-to-last layer of BERT and the word embedding layer of NMT. We also explored the fusion with different NMT layer but achieved no sustained gains. For asymptotic distillation, the balance coefficient $\alpha$ is set to 0.9 in Eq. (5). We compare our approach with MultiCol approach which pretrained the encoders with NMT model and merges the outputs of a single combined representation. The performance lags behind our best performance. And with only Asymptotic Distillation we still outperform MultiCol without additional parameters.

\section{Results and Analysis}

The results on English-German and English-French translation are presented in Table 1. We compare CTNMT with various other systems including Transformer and previous stateof-the-art pre-trained LM enhanced model. As observed by Edunov, Baevski, and Auli (2019), Transformer big model with fine-tuning approach even falls behind the baseline. They then freeze the LM parameters during fine-tuning and achieve a few gains over the strong transformer big model. This is consistent with our intuition that fine-tuning on the large dataset may lead to degradation of the performance. In CTNMT, we first evaluate the effectiveness of the proposed three strategies respectively. Clearly, these method achieves almost 2 BLEU score improvement over the state-of-the-art on the English-German task for the base network. In the case of the larger English-French task, we obtain 1.2 BLEU improvement for the base model. In the case of the EnglishChinese task, we obtain 1.6 BLEU improvement for the baseline model. More importantly, the combination of these strategies finally gets an improvement over the best single strategy with roughly $0.5 \mathrm{BLEU}$ score. We will then give a detailed analysis as followings.

\section{Encoder v.s. Decoder}

\begin{tabular}{c|c}
\hline Models & En $\rightarrow$ De BLEU \\
\hline BERT Enc & 29.2 \\
BERT Dec & 26.1 \\
GPT-2 Enc & 27.7 \\
GPT-2 Dec & 27.4 \\
\hline
\end{tabular}

Table 2: Ablation of asymptotic distillation on the encoder and the decoder of NMT.

As shown in Table 2, pre-trained language model representations are most effective when supervised on the encoder part but less effective on the decoder part. As BERT contains bidirectional information, pre-training decoder may lead inconsistencies between the training and the inference. The GPT-2 Transformer uses constrained selfattention where every token can only attend to context to its left, thus it is natural to introduce GPT-2 to the NMT decoder. While there are still no more significant gains obtained in our experiments. One possible reason is that the decoder is not a typical language model, which contains the information from source attention. We will leave this issue in the future study.

\section{BERT v.s. GPT-2}

We compare BERT with GPT-2(Radford et al. 2019; 2018) on WMT 2014 English-German corpus. As shown in Table 2, BERT added encoder works better than GPT-2. The experiments suggest that bidirectional information plays an important role in the encoder of NMT models. While for the decoder part, GPT-2 is a more priority choice. In the following part, we choose BERT as the pre-trained LM and apply only for the encoder part.

\section{About asymptotic distillation}

We conduct experiments on the performance of asymptotic distillation model on different amounts of training data. The results are listed in table 3 . The experiments is in line with our intuition that with the increasing training data, 


\begin{tabular}{c|ccc}
\hline & Transformer & Fine-tuning & AD \\
\hline $900 \mathrm{~K}$ & 16.6 & 19.8 & 20.2 \\
$1,800 \mathrm{~K}$ & 22.5 & 24.6 & 25.1 \\
$2,700 \mathrm{~K}$ & 24.5 & 25.2 & 26.9 \\
$3,600 \mathrm{~K}$ & 26.2 & 26.8 & 28.4 \\
\hline $4,500 \mathrm{~K}$ & 27.2 & 27.8 & 29.2 \\
\hline
\end{tabular}

Table 3: Ablation of using different data size with asymptotic distillation.

the gains of fine-tuning will gradually diminish. While with the asymptotic distillation, we achieve continuous improvements.

\section{About dynamic switch}

\begin{tabular}{l|c}
\hline \multicolumn{1}{c|}{ Models } & En $\rightarrow$ De BLEU \\
\hline Transformer & 27.2 \\
Encoder w/o BERT init & - \\
BERT Feature w/o Encoder & 25.2 \\
BERT + Encoder & 28.5 \\
BERT @ Encoder & 29.4 \\
\hline
\end{tabular}

Table 4: Results on WMT14 English-German with different feeding strategies. '+' indicates average pooling and '@ ' indicates dynamic switch.

We then compare different ways to combine the embedding vector and the BERT features which will be fed into the Transformer encoder. In Table 4, we first conduct experiments with 24 layer encoder without BERT pre-training to figure out if the improvements comes from the additional parameters. The model cannot get meaningful results due to gradient vanish problem. This also suggests that good initialization help to train the deep model. In the third row, we replace the NMT encoder with BERT and keep the BERT parameters frozen during fine-tuning, the performance lags behind the baseline, which indicates the importance of the original NMT encoder. According to the above experimental results, we combine both the BERT and NMT encoder. In the fourth row, the average pooling method obtains a gain of 1.3 BLEU score over the baseline model showing the power of combination. Finally, the dynamic switch strategy keep the balance between BERT and NMT and achieve a substantial improvement of 0.9 BLEU score over the average pooling approach.

\section{About rate-scheduled learning}

In Table 5, we evaluate the fine-tuning based strategies on WMT 2014 English-German corpus. For $\eta^{l m}=1$, the model draws back to the traditional fine-tuning approach, while for $\eta^{l m}=0$, the model is exactly the feature-based approach. We mainly compare two settings for rate-scheduled learning models: 1) we fix $\eta^{l m}=0.01$, a small constant update weight; 2) we follow slanted triangular learning rates policy in Eq.(10) to dynamically apply the $\eta^{l m}$ to the SGD update. The results show that slanted triangular learning

\begin{tabular}{l|c}
\hline \multicolumn{1}{c|}{ Models } & En $\rightarrow$ De BLEU \\
\hline$\eta^{l m}=1$ & 27.7 \\
$\eta^{l m}=0.01$ & 29.0 \\
$\eta^{l m}=\rho \eta^{n m t}$ & 29.7 \\
$\eta^{l m}=0$ & 28.4 \\
\hline
\end{tabular}

Table 5: Results on WMT14 English-German with ratescheduled learning. $\eta^{l m}=1$ indicates the fine-tuning approach and $\eta^{l m}=0$ indicates the frozen feature-based approach.

rates policy is a more promising strategy for fine-tuning models. We find that changing $\eta^{l m}$ during the training phase provides better results than fixed values with a similar or even smaller number of epochs. The conclusion is in line with (Smith 2017).

\section{About BERT layers}

\begin{tabular}{l|c}
\hline \multicolumn{1}{c|}{ Models } & En $\rightarrow$ De BLEU \\
\hline Last Hidden & 28.4 \\
Second-to-Last Hidden & 29.2 \\
Third-to-Last Hidden & 29.2 \\
Fourth-to-Last Hidden & 29.2 \\
\hline
\end{tabular}

Table 6: Results on WMT14 English-German with different layers of BERT.

We implement asymptotic distilling by applying auxiliary L2 loss between a specific NMT encoder layer and a specific BERT layer. In our experiments, we add the supervision signal to the $3^{\text {th }}$ encoder layer. In Table 6 , it is interesting to find that the second-to-last layer of BERT works significantly better than the last hidden state. Intuitively, the last layer of the pre-trained LM model is impacted by the LM target-related objective (i.e. masked language model and next sentence prediction) during pre-training and hence the last layer is biased to the LM targets.

\section{Related work}

\section{Unsupervised pre-training of LMs}

Unsupervised pre-training or transfer learning has been applied in a variety of areas where researchers identified synergistic relationships between independently collected datasets. Dahl et al. (2012) is the pioneering work that found pre-training with deep belief networks improved feedforward acoustic models. Natural language processing leverages representations learned from unsupervised word embedding (Mikolov et al. 2013; Pennington, Socher, and Manning 2014) to improve performance on supervised tasks, such as named entity recognition, POS tagging semantic role labelling, classification, and sentiment analysis (Collobert et al. 2011; Socher et al. 2013; Wang and Zheng 2015; Tan et al. 2018). The word embedding approaches have been 
generalized to coarser granularities as well, such as sentence embedding (Kiros et al. 2015; Le and Mikolov 2014).

Recently, Peters et al. (2018) introduced ELMo, an approach for learning universal, deep contextualized representations using bidirectional language models. They achieved large improvements on six different NLP tasks. A recent trend in transfer learning from language models (LMs) is to pre-train an LM model on an LM objective and then fine-tune on the supervised downstream task. OpenAI GPT2 (Radford et al. 2019; 2018) achieved remarkable results in many sentence level tasks from the GLUE benchmark. Devlin et al. (2018) introduce pre-trained BERT representations which can be fine-tuned with just one additional output layer, achieving the state-of-the-art performance. Our work builds on top of the pre-training of LMs. To make the work reproducible, we choose the public BERT ${ }^{4}$ and GPT- $2^{5}$ as the strong baseline.

\section{Pre-training for NMT}

A prominent line of work is to transfer the knowledge from resource-rich tasks to the target resource-poor task. Qi et al. (2018) investigates the pre-trained word embedding for NMT model and shows desirable performance on resourcepoor languages or domains. Ramachandran, Liu, and Le (2017) presents a general unsupervised learning method to improve the accuracy of sequence to sequence (seq2seq) models. In their method, the weights of the encoder and the decoder of a seq2seq model are initialized with the pretrained weights of two LMs and then fine-tuned with the parallel corpus.

There have also been works on using data from multiple language pairs in NMT to improve performance. Gu et al.; Zoph et al. $(2018 ; 2016)$ showed that sharing a source encoder for one language helps performance when using different target decoders for different languages. They then fine-tuned the shared parameters to show improvements in a poorer resource setting.

Perhaps most closely related to our method is the work by Lample and Conneau; Edunov, Baevski, and Auli (2019; 2019) who feeds the last layer of ELMo or BERT to the encoder of NMT model. While following the same spirit, there are a few key differences between our work and theirs. One is that we are the first to leverage ssymptotic distillation to transfer the pre-training information to NMT model and empirically prove its effectiveness on truly large amounts of training data (e.g. tens of millions). Additionally, the aforementioned previous works directly feed the LM to NMT encoder, ignoring the benefit of the traditional NMT encoder features. We extend this approach with dynamic switch and rate-scheduled learning strategy to overcome the catastrophic forgetting problem. we finally incorporate the three strategies and find they can complement each other and achieve the state-of-the-art on the benchmark WMT dataset.

\footnotetext{
${ }^{4}$ https://github.com/google-research/bert

${ }^{5}$ https://github.com/openai/gpt-2/
}

\section{Conclusion}

We propose CTNMT, an effective, simple, and efficient transfer learning method for neural machine translation that can be also applied to other NLP tasks. Our conclusions have practical effects on the recommendations for how to effectively integrate pre-trained models in NMT: 1) Adding pre-trained LMs to the encoder is more effective than the decoder network. 2) Employing CTNMT addresses the catastrophic forgetting problem suffered by pre-training for NMT. 3) Pre-training distillation is a good choice with nice performance for computational resource constrained scenarios. While the empirical results are strong, CTNMT surpasses those previous pre-training approaches by 1.4 BLEU score on the WMT English-German benchmark dataset. On the other two large datasets, our method still achieves remarkable performance.

\section{References}

Bojanowski, P.; Grave, E.; Joulin, A.; and Mikolov, T. 2017. Enriching word vectors with subword information. Transactions of the Association for Computational Linguistics 5:135-146.

Chen, M. X.; Firat, O.; Bapna, A.; Johnson, M.; Macherey, W.; Foster, G.; Jones, L.; Schuster, M.; Shazeer, N.; Parmar, N.; Vaswani, A.; Uszkoreit, J.; Kaiser, L.; Chen, Z.; Wu, Y.; and Hughes, M. 2018. The best of both worlds: Combining recent advances in neural machine translation. In $A C L$.

Chung, J.; Gülçehre, Ç.; Cho, K.; and Bengio, Y. 2014. Empirical evaluation of gated recurrent neural networks on sequence modeling. CoRR abs/1412.3555.

Collobert, R.; Weston, J.; Bottou, L.; Karlen, M.; Kavukcuoglu, K.; and Kuksa, P. P. 2011. Natural language processing (almost) from scratch. Journal of Machine Learning Research 12:2493-2537.

Dahl, G. E.; Yu, D.; Deng, L.; and Acero, A. 2012. Context-dependent pre-trained deep neural networks for large-vocabulary speech recognition. IEEE Transactions on Audio, Speech, and Language Processing 20(1):30-42.

Devlin, J.; Chang, M.-W.; Lee, K.; and Toutanova, K. 2018. Bert: Pre-training of deep bidirectional transformers for language understanding. arXiv preprint arXiv:1810.04805.

Edunov, S.; Baevski, A.; and Auli, M. 2019. Pre-trained language model representations for language generation. CoRR abs/1903.09722.

Goodfellow, I. J.; Mirza, M.; Xiao, D.; Courville, A.; and Bengio, Y. 2013. An Empirical Investigation of Catastrophic Forgetting in Gradient-Based Neural Networks. arXiv eprints arXiv:1312.6211.

Gu, J.; Hassan, H.; Devlin, J.; and Li, V. O. 2018. Universal neural machine translation for extremely low resource languages. In Proceedings of the 2018 Conference of the North American Chapter of the Association for Computational Linguistics: Human Language Technologies, Volume 1 (Long Papers), 344-354. New Orleans, Louisiana: Association for Computational Linguistics. 
Howard, J., and Ruder, S. 2018. Universal language model fine-tuning for text classification. In Proceedings of the 56th Annual Meeting of the Association for Computational Linguistics (Volume 1: Long Papers), 328-339. Melbourne, Australia: Association for Computational Linguistics.

Kiros, R.; Zhu, Y.; Salakhutdinov, R.; Zemel, R. S.; Torralba, A.; Urtasun, R.; and Fidler, S. 2015. Skip-thought vectors. neural information processing systems 3294-3302.

Lample, G., and Conneau, A. 2019. Cross-lingual language model pretraining. CoRR abs/1901.07291.

Le, Q., and Mikolov, T. 2014. Distributed representations of sentences and documents. In Xing, E. P., and Jebara, T., eds., Proceedings of the 31st International Conference on Machine Learning, volume 32 of Proceedings of Machine Learning Research, 1188-1196. Bejing, China: PMLR.

Mikolov, T.; Sutskever, I.; Chen, K.; Corrado, G. S.; and Dean, J. 2013. Distributed representations of words and phrases and their compositionality. neural information processing systems 3111-3119.

Papineni, K.; Roukos, S.; Ward, T.; and Zhu, W.-J. 2002. Bleu: a method for automatic evaluation of machine translation. In Proceedings of the 40th annual meeting on association for computational linguistics, 311-318. Association for Computational Linguistics.

Pennington, J.; Socher, R.; and Manning, C. 2014. Glove: Global vectors for word representation. In Proceedings of the 2014 Conference on Empirical Methods in Natural Language Processing (EMNLP), 1532-1543. Doha, Qatar: Association for Computational Linguistics.

Pereyra, G.; Tucker, G.; Chorowski, J.; Kaiser, Ł.; and Hinton, G. 2017. Regularizing neural networks by penalizing confident output distributions. arXiv preprint arXiv:1701.06548.

Peters, M.; Neumann, M.; Iyyer, M.; Gardner, M.; Clark, C.; Lee, K.; and Zettlemoyer, L. 2018. Deep contextualized word representations. In Proceedings of the 2018 Conference of the North American Chapter of the Association for Computational Linguistics: Human Language Technologies, Volume 1 (Long Papers), 2227-2237. New Orleans, Louisiana: Association for Computational Linguistics.

Qi, Y.; Sachan, D.; Felix, M.; Padmanabhan, S.; and Neubig, G. 2018. When and why are pre-trained word embeddings useful for neural machine translation? In Proceedings of the 2018 Conference of the North American Chapter of the Association for Computational Linguistics: Human Language Technologies, Volume 2 (Short Papers), 529-535. New Orleans, Louisiana: Association for Computational Linguistics.

Radford, A.; Narasimhan, K.; Salimans, T.; and Sutskever, I. 2018. Improving language understanding by generative pre-training.

Radford, A.; Wu, J.; Child, R.; Luan, D.; Amodei, D.; and Sutskever, I. 2019. Language models are unsupervised multitask learners.

Ramachandran, P.; Liu, P.; and Le, Q. 2017. Unsupervised pretraining for sequence to sequence learning. In Proceed- ings of the 2017 Conference on Empirical Methods in Natural Language Processing, 383-391. Copenhagen, Denmark: Association for Computational Linguistics.

Smith, L. N. 2017. Cyclical learning rates for training neural networks. In WACV, 464-472. IEEE Computer Society.

Socher, R.; Perelygin, A.; Wu, J. Y.; Chuang, J.; Manning, C. D.; Ng, A. Y.; and Potts, C. 2013. Recursive deep models for semantic compositionality over a sentiment treebank. 1631-1642.

Tan, Z.; Wang, M.; Xie, J.; Chen, Y.; and Shi, X. 2018. Deep semantic role labeling with self-attention. national conference on artificial intelligence 4929-4936.

Vaswani, A.; Shazeer, N.; Parmar, N.; Uszkoreit, J.; Jones, L.; Gomez, A. N.; Kaiser, L. u.; and Polosukhin, I. 2017a. Attention is all you need. In Guyon, I.; Luxburg, U. V.; Bengio, S.; Wallach, H.; Fergus, R.; Vishwanathan, S.; and Garnett, R., eds., Advances in Neural Information Processing Systems 30. Curran Associates, Inc. 5998-6008.

Vaswani, A.; Shazeer, N.; Parmar, N.; Uszkoreit, J.; Jones, L.; Gomez, A. N.; Kaiser, L.; and Polosukhin, I. 2017b. Attention is all you need. neural information processing systems 5998-6008.

Wang, D., and Zheng, T. F. 2015. Transfer learning for speech and language processing. asia pacific signal and information processing association annual summit and conference 1225-1237.

Zoph, B.; Yuret, D.; May, J.; and Knight, K. 2016. Transfer learning for low-resource neural machine translation. In Proceedings of the 2016 Conference on Empirical Methods in Natural Language Processing, 1568-1575. Austin, Texas: Association for Computational Linguistics. 